\title{
Function, structure and quality of striated muscles in the lower extremities in patients with late onset Pompe Disease - an MRI study
}

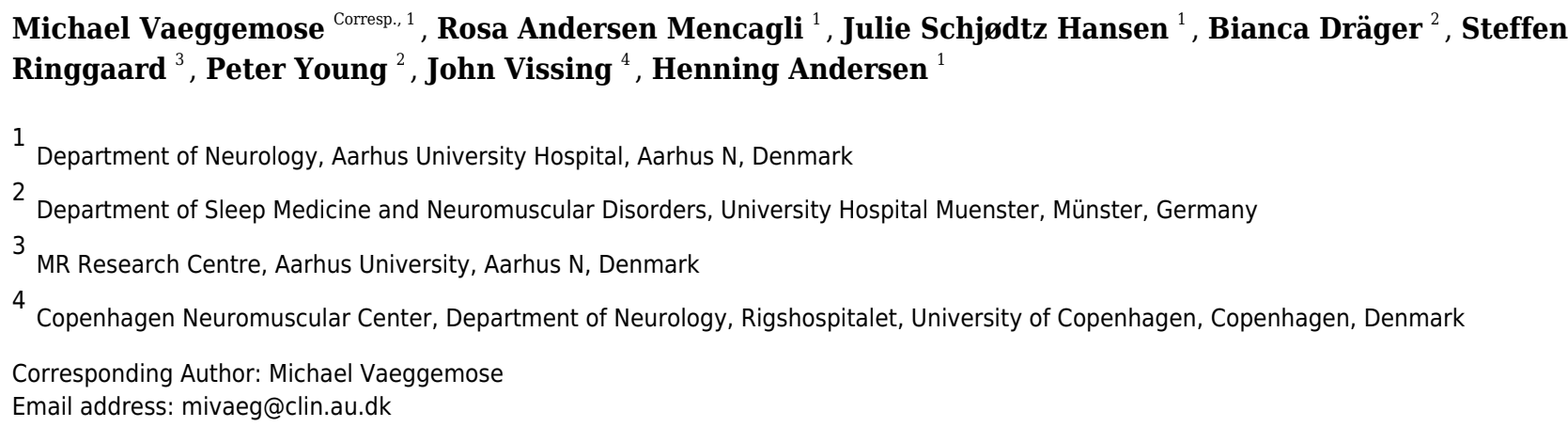

Background: Pompe Disease (PD) is a rare inherited metabolic myopathy, caused by lysosomal- $\alpha$-glucosidase (GAA) deficiency, which leads to glycogen accumulation within the lysosomes, resulting in cellular and tissue damage. Due to the emergence of a disease modifying treatment with recombinant GAA there has been a large increase in studies of late onset Pompe Disease (LOPD) during the last decade. Methods: The present study evaluates muscle quality in 10 patients with LOPD receiving treatment with enzyme replacement therapy and in 10 age and gender matched healthy controls applying $T_{1}$ weighted DIXON MR imaging and isokinetic dynamometry. Muscle quality was determined by muscle strength in relation to muscle size (contractile cross-sectional area, CSA) and to muscle quality (fat fraction). A follow-up evaluation of the patients was performed after 8-12 months. Patient evaluations also included: six-minute walking test (6MWT), forced vital capacity, manual muscle testing and SF-36 questionnaire. Results: Fat fraction of knee flexors $(0.15$ vs $0.07, p<0.05)$ and hip muscles $(0.11$ vs $0.07, p<0.05)$ were higher in patients than controls. In patients, contractile CSA correlated with muscle strength (knee flexors: $r=0.86$, knee extensors: $r=0.88$, hip extensors: $r=0.83, p<0.05$ ). No correlation was found between fat fraction and muscle strength. The fat fraction of thigh muscles did not correlate with scores from the clinical tests nor did it correlate with the 6MWT. During follow-up, the contractile CSA of the knee extensors increased by $2 \%$. No other statistically significant change was observed. Quantitative MRI reflects muscle function in patients with LOPD, but larger long-term studies are needed to evaluate its utility in detecting changes over time. 
1 Function, structure and quality of striated muscles in the lower

2

3

4

5

6

7

8

9

10

11

12

13

14

15

16

17

18

19

20

21

22

23

24

25

26

27

28

29

30

31

32

33

34

35 extremities in patients with late onset Pompe Disease - an MRI study

\author{
M. Vaeggemose ${ }^{1}$, R. A. Mencagli ${ }^{1}$, J.S. Hansen ${ }^{1}$, B. Dräger ${ }^{2}$, S. Ringgaard ${ }^{3}$, P. Young ${ }^{2}$, J. \\ Vissing ${ }^{4}, \mathrm{H}$. Andersen ${ }^{1}$
}

1

1. Department of Neurology, Aarhus University Hospital, Aarhus, Denmark

2. Department of Sleep Medicine and Neuromuscular Disorders, University of Münster, Münster, Germany

4 3. MR Research Centre, Aarhus University, Aarhus, Denmark

5

4. Copenhagen Neuromuscular Center, Department of Neurology, Rigshospitalet, University of Copenhagen, Denmark

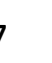

8

(1)

1

3

2 Corresponding Author:

Michael Vaeggemose ${ }^{1}$

4 Palle Juul-Jensens Blvd. 99, 8200, Aarhus, Denmark

35 Email address: mivaeg@clin.au.dk 


\section{ABSTRACT}

37

\section{Background}

39 Pompe Disease (PD) is a rare inherited metabolic myopathy, caused by lysosomal- $\alpha$-glucosidase (GAA) deficiency, which leads to glycogen accumulation within the lysosomes, resulting in cellular and tissue damage. Due to the emergence of a disease modifying treatment with recombinant GAA there has been a large increase in studies of late onset Pompe Disease (LOPD) during the 43 last decade.

44

45

\section{3} 65

\section{Methods}

The present study evaluates muscle quality in 10 patients with LOPD receiving treatment with enzyme replacement therapy and in 10 age and gender matched healthy controls applying $\mathrm{T}_{1^{-}}$ weighted DIXON MR imaging and isokinetic dynamometry. Muscle quality was determined by muscle strength in relation to muscle size (contractile cross-sectional area, CSA) and to muscle quality (fat fraction). A follow-up evaluation of the patients was performed after 8-12 months. Patient evaluations also included: six-minute walking test (6MWT), forced vital capacity, manual muscle testing and SF-36 questionnaire.

\section{Results}

Fat fraction of knee flexors $(0.15$ vs $0.07, p<0.05)$ and hip muscles $(0.11$ vs $0.07, p<0.05)$ were higher in patients than controls. In patients, contractile CSA correlated with muscle strength (knee flexors: $r=0.86$, knee extensors: $r=0.88$, hip extensors: $r=0.83, p<0.05$ ). No correlation was found between fat fraction and muscle strength. The fat fraction of thigh muscles did not correlate with scores from the clinical tests nor did it correlate with the 6MWT. During followup, the contractile CSA of the knee extensors increased by $2 \%$. No other statistically significant change was observed.

Quantitative MRI reflects muscle function in patients with LOPD, but larger long-term studies are needed to evaluate its utility in detecting changes over time.

(1)




\section{1. Introduction}

68 Pompe Disease (PD) is a rare inherited metabolic myopathy, caused by lysosomal- $\alpha$-glucosidase 69 (GAA) deficiency, which leads to glycogen accumulation within the lysosomes, resulting in cellular and tissue damage [1]. PD presents clinically in two forms, the infantile Pompe with no residual enzyme activity [2] [3], and the less severe late onset Pompe Disease (LOPD). LOPD may be symptomatic from young age to adulthood and is characterized by some residual activity of GAA. The skeletal muscles gradually degenerate with accumulating fat infiltration [4]. Skeletal muscle dysfunction results in muscle weakness, fatigue and respiratory insufficiency. With disease progression, patients may become wheelchair-bound and need ventilator support [3] [5], and have reduced life expectancy [6].

Due to the emergence of a disease modifying treatment with recombinant GAA there has been a large increase in studies of LOPD during the last decade, which have expanded our knowledge about LOPD as a broader phenotype of a multisystemic disease [7]. Treatment with recombinant GAA, also known as enzyme replacement therapy (ERT), has been available for LOPD since 2006, and new drugs are under development. Enzyme replacement therapy (ERT) has a wellestablished but moderate effect in most patients, however, the response to the treatment varies [8]. In a slowly progressing disease such as LOPD, monitoring the effect of a treatment is challenging, particularly as the treatment merely attenuates the disease progression rather than improving the functional capacity. The European Guidelines [9] recommend the following three endpoints for monitoring the disease: I. Functional tests; primarily the 6 minutes walking test (6MWT) and the four timed tests (walking 10 metres, climbing four steps, standing up from supine position and standing up from a chair). II. Forced vital capacity (FVC) in sitting and lying position and maximal inspiratory/expiratory pressure (MIP/MEP). III. Muscle strength assessed by manual muscle test (MRC).

However, the recommended tests are susceptible to variation in performance, due to patient dependence (lack of motivation and concentration, fatigue, pain, learning effects, and inherent variations in the measurements). This impedes detection of small changes in the patient's performance, especially over short periods of time and in small patient populations. Thus, there is still the need for sensitive and reliable objective methods to monitor LOPD patients. 
96

97

98

100

101

102

103

104

105

106

107

108

109

110

111

112

113

114

115

116

117

118

119

120

121

122

Magnetic resonance imaging (MRI) is a promising tool as it enables identification and quantification of fat replacement in skeletal muscles. In recent years, there has been a growing interest in the clinical use of MRI of skeletal muscles in neuromuscular disorders [10] [11] [12] [13]. Studies have reported close correlations between MRI findings and clinical tests although semi quantitative methods were primarily used. Interestingly, quantitative MRI has also been shown to enable detection of subclinical changes in fat fraction over time [14]. However, results are difficult to compare as some MRI studies have not included quantitative techniques to determine strength of individual muscle groups [15], have only performed cross sectional studies [16] or have only included a few muscle groups [17].

Therefore, the aims of this study were to evaluate the quality of a high number of muscles in patients with LOPD by determining the correlation between isokinetic muscle strength and the contractile cross-sectional area (CCSA). Furthermore, to evaluate whether changes in fat infiltration and the contractile cross sectional determined by MRI in the lower extremity corresponds to changes in disease severity during a shorter follow up period.

\section{Materials and methods}

\subsection{Population}

Between September 2015 and May 2017, all 11 Danish patients diagnosed with LOPD were invited to participate in the study. Eight accepted participation, one did not answer, and two declined participation due to the need for respiratory support during the MR scan. To increase the statistical power of the study, two patients were recruited from the Department of Sleep Medicine and Neuromuscular Disorders at Münster University Hospital, Germany, resulting in a total of 10 patients with a genetically confirmed diagnosis of LOPD (Figure 1). All patients were examined at day 1 and again after 8 months, except 3 patients, who were diagnosed during the study period. They were examined after 12 months to experience a complete 8 months of drug administration for study comparison. Nine patients received alglucosidase alfa and 1 patient received avalglucosidase alfa (neoGAA). 
123 For comparison, 10 healthy controls matched for age, sex, height and weight were recruited and 124 examined. Controls were recruited through advertisement in a local newspaper, on an online 125 webpage for healthy controls and through public posters.

126 Exclusion criteria where: Age < 18 years, contraindications to MRI (metal in the body, pacemaker, 127 pregnancy, and claustrophobia), cancer and other serious medical conditions and abuse of 128 alcohol. Healthy control subjects were only examined by isokinetic dynamometry and MRI. All 129 participants gave informed signed consent and were examined at the Department of Neurology, 130 Aarhus University Hospital, Denmark. The study was approved by the Danish National 131 Committee On Health Research Ethics (application no. 49089) and by the Danish Data Protection 132 Agency. The study was registered at ClinicalTrials.gov (identifier: NCT02708784).

134 2.2. MRI acquisition

135 MR scanning was performed at the MR Centre at Aarhus University Hospital using a 3-T Siemens 136 Skyra Magnetom (Siemens AG, Erlangen, Germany). The scan protocol consisted of a T1137 weighted, 3-point gradient echo DIXON sequence (400 slices of $3 \mathrm{~mm}$ thickness, 20\% gap, TR 5.31 $138 \mathrm{~ms}$, TE $2.46 \mathrm{~ms}$, flip angle $9^{\circ}$, FOV 500, in-plane resolution $1 \times 1 \mathrm{~mm}$ ) acquiring images in a total 139 scan time of 40 minutes. Participants were lying in a feet-first supine position and MR images 140 were recorded from the ankle (lateral malleolus) to the trochanter major with the use of a 36 141 channel peripheral angio coil (Siemens AG, Erlangen, Germany) and from the trochanter major 142 to L1 with a 18 channel body matrix coil (Siemens AG, Erlangen, Germany).

\subsection{MRI data analysis}

145 MR images were analysed using Osirix (Version 6.5.2, Pixmeo SARL, Switzerland). Muscle 146 segmentation was done manually by segmenting regions of interest (ROI) in the T1-weigthed 147 opposed-phase images, recorded with the Dixon sequence. The following 12 muscles were 148 segmented at the non-dominant thigh: biceps femoris caput longum muscle, semimembranosus 149 muscle, semitendinosus muscle, rectus femoris muscle, vastus medialis muscle, vastus lateralis 150 muscle, vastus intermedius muscle, sartorius muscle, gluteus maximus muscle, adductor magnus 
151 muscle, adductor longus muscle and iliopsoas muscle. To determine the muscle and fat fractions

152 the ROIs were applied to the corresponding fat and water images calculated from the Dixon

153 sequence. Based on the signal intensity (SI) the muscle fat fraction was calculated as the signal

154 intensity from the fat image divided with the combined fat and water signal intensity from a given

155 ROI (FF = SI(fat) / [SI(fat) + SI(water) $])$.

156 The fat fraction of the knee flexors was calculated as the mean fat faction from the following 157 muscles: biceps femoris caput longum, semimembranosus and semitendinosus; the fat fraction

158 of the knee extensors was calculated including the muscles: sartorius, rectus femoris, vastus 159 lateralis, vastus intermedius and vastus medialis.

160 The contractile cross-sectional area (CSA) was determined as the cross-sectional muscle area 161 subtracted the fat infiltrated muscle area (CSA = Area * [1 - FF]) similar to the method described 162 by Carlier et al [11]).

163 To reduce bias in segmentation of muscles close to ligaments five cross sections of each muscle 164 were segmented with respect to the centre slice between two bone fix points illustrated by figure 165 2. For the knee extensors, the knee flexors, the adductor magnus muscle and the adductor longus 166 muscle, the slices chosen corresponded to the middle five slices between the trochanter major 167 on the femur and the upper edge of the patella. The five slices analysed to evaluate the gluteus 168 maximus muscle and the iliopsoas muscle corresponded to the most proximal part of the femur 169 bone. The slices chosen correspond to the slice where the caput femoris first appears together 170 with the two nearby proximal and distal slices. Although, this results in segmentation of the most 171 distal part of the iliopsoas muscle, which is not representative for the whole muscle, this 172 segmentation was chosen as respiration and bowel artefacts was present in images of the $\mathrm{m}$. 173 iliopsoas more proximally. The vastus intermedius and the vastus lateralis muscles are difficult to 174 discriminate and therefore the muscles were analysed together, as proposed by Barnouin et al. 175 [18]. The short head on the biceps femoris muscle was not analysed, as the muscle was not visible 176 in all subjects on the chosen slices. Due to respiratory difficulties patient 1 was supported with 177 cushions enabling only MRI images from the ankle to the upper knee. 


\section{2.4. Isokinetic muscle strength}

180 Isokinetic muscle strength was assessed by dynamometry using a Biodex System 3 PRO 181 dynamometer (Biodex Medical System, NY USA; software version 3.1) as described earlier [19].

182 The protocol included eight repetitions for each movement and standardized instructions before 183 and during the tests. The maximal peak torque was used for further analysis together with the 184 percentage of expected strength, calculated according to Harbo et al. In case of a coefficient of

185

186

187

188

189

190

191

192

193

194

195

196

197

198

199

200

201

202

203

204 variation $>15 \%$, the test was repeated. Maximal muscle strength was determined for the flexors and extensors of the ankle, knee, hip, elbow and wrist, together with shoulder abduction and adduction. Patient 1 was unable to perform hip flexion and extension, due to need of respiratory support in supine position. Patients 3, 6 and 9 were not able to perform all the tests due to muscle weakness and pain. Patient 3 could only perform hip flexion, shoulder abduction, elbow flexion and hand flexion and extension. Patient 6 was unable to perform hip flexion and extension. Patient 9 was unable to perform hip flexion, shoulder abduction and elbow flexion.

\subsection{Clinical measurements}

Muscle strength was determined by manual muscle testing by a trained neurologist (HA) and graded as follows ( $0=$ full strength, $1=25 \%$ reduced strength, $2=50 \%$ reduced strength, $3=75 \%$ reduced strength and $4=100 \%$ reduced strength). Exercise capacity was assessed by the 6 minutes walking test (6MWT). Vital capacity was determined according to the European Respiratory Society Guidelines [20] using a spirometry system in both sitting and supine position. The highest measurement out of three consecutive tests, defined as \% of expected forced vital capacity (FVC) in litres, was used in further analyses. Perceived quality of life was established according to the Danish version of the 36-Item Short Form (SF-36) questionnaire.

Finally, the patients were ranked according to each of these clinical measurements, and a summed rank-score was obtained as a measure of the combined disease severity status based on the 10 participating LOPD subjects. 
206

207

208

209

210

211

212

213

214

215

216

217

218

219

220

221

222

223

224

225

226

227

228

229

230

\subsection{Statistical analysis}

Muscle strength, fat fraction and contractile CSA in LOPD patients were compared to healthy controls by paired t-test and Wilcoxon signed rank test. Correlations between muscle strength and muscle quality and between total fat fraction and clinical tests were tested by Spearman's correlation and linear regression both for patients and controls. Follow-up data were analysed using paired t-test. Statistical significance was determined with a p-value of 0.05 or less.

\section{Results}

Demographics of the ten patients with LOPD and ten healthy matched controls are shown in table 1. Furthermore, the clinical characteristics with gene mutations of the patient population are described in table 2.

\subsection{Contractile cross-sectional area and fat fraction}

Contractile CSA of the knee flexors, the knee extensors, the gluteus maximus muscle and the adductor magnus muscle was reduced in the patients compared to their healthy controls (table 3). However, no statistical difference was found for the iliopsoas muscle and the adductor longus muscle. In all muscles examined, patients had a higher fat fraction, apart from the knee extensors (table 3 and figure 3). In the lower limbs, patients had reduced strength of knee extensors, hip flexors and hip extensors. In the upper limb, strength was reduced for shoulder adductors and elbow flexors only (table 3).

\subsubsection{Muscle quality}

Close correlations were found between the contractile CSA and muscle strength of the knee extensors in both healthy controls and patients (table 4).

In patients, the contractile CSA of the knee flexors and hip extensors and the fat fraction of the knee flexors also correlated with muscle strength. 
231 No correlation was found between the total fat fraction and the summed rank-score determined

232 from the clinical tests, nor between the total fat fraction and the 6MWT (table 4).

\section{$234 \quad 3.1 .2$ Longitudinal analysis}

235 During the follow-up period no change was observed at any of the clinical tests (table 5).

236 Contractile CSA of the knee extensors increased slightly. The other contractile CSA and the fat

237 fractions remained stable (tables 6 and 7).

238 No correlation was found between the change in expected muscle strength and the change in 239 the total fat fraction, neither between the relative changes in $\%$ of total expected muscle strength 240 and total fat fraction or total CSA (figure 4).

\section{4. Discussion}

242 Fat fraction determined by MRI was higher in patients with LOPD as compared to healthy 243 controls. The replacement of muscle by fat followed a selective pattern, with more involvement 244 of the adductor magnus muscle, the muscles at the pelvic girdle and the hamstrings combined 245 with selective sparing of the short head of the biceps femoris muscle. This finding is in line with 246 previous studies [4] [21]. In patients with more advanced disease there was fat replacement in 247 knee extensors also, however, there was selective sparing of the rectus femoris, sartorius and 248 gracilis muscles. Although the fat fraction of the healthy controls was lower than the patients', 249 the controls had a surprisingly high fat fraction compared to earlier studies [22] [23] [24]. This 250 could be due to inclusion of physically less active subjects as in earlier studies.

252 Contractile CSA of the knee extensors closely correlated with isokinetic strength both in patients 253 and controls. In patients, close correlations were also found between the contractile CSA of the 254 knee flexors and hip extensors when compared to muscle strength. However, no correlation was 255 found for hip flexion, which may due to a smaller muscle size. The iliopsoas muscle was analyzed only very distally due to bowel and respiration artefacts, which may explain the lack of correlation between contractile CSA and muscle strength. Pichiecchio et al reported close correlations 
258 between muscle volume and strength, however, they only used semiquantitative strength 259 measurements [21]. Similar close correlations between strength and contractile CSA have also 260 been described in other neuromuscular disorders supporting the sensitivity of the MRI method $261[23][25]$.

262 In contrast to previous studies of patients with LOPD and other neuromuscular disorders [21] 263 [23] [24] [16] [26] [27] [28] [15] [29], we did not find any correlation between fat fraction and 264 muscle strength, with the exception of the knee flexors. In the studies by Figueorora-Bonaparte 265 et al and Khan et al there was a very close relationship between motor function tests including

266

267

268

269

270

271 272 did not report on the hip extensors.

273 Unexpectedly, in our healthy control group we found only strong correlations for the contractile 274 CSA of the knee extensors and hip flexors. This may be explained by a low number of subjects

275

276

277 278

279 280

281

282

283

284 285 muscle strength and fat fraction. The discrepancy with our study may be due to more severely affected patients and a larger study population compared to our study. Interestingly, our findings are similar to those of Bonaparte et al. [16] who also studied muscles in patients with LOPD applying quantitative muscle strength measurements and $\mathrm{MRI}$, and only found strong correlations for knee flexors $(r=0.70)$, while the correlation for knee extensors was weaker $(r=0.63)$. In line with our findings they reported no correlation for the hip flexors whereas they

included. In healthy subjects there is a great variation in strength to CSA ratio [30], and this may explain our findings considering the larger variability of isokinetic testing of the knee flexors and the hip flexors and extensors as compared to the knee extensors.

In our LOPD patients, the mean fat fraction did not correlate with the overall clinical status, defined from the sum-rank score of the rankings from the clinical tests (vital capacity, SF-36, manual muscle testing and 6MWT). This is not surprising since some studies show a close correlation between fat fraction and functional tests, such as the 6MWT and "time to climb up/go down 4 stairs" [23] [16] [31] [32] [15], while other studies did not find any correlation [33] [32]. Such inconsistent findings may be explained by the high clinical heterogeneity of patients with LOPD and similar diseases [34]. Some patients have substantial weakness, pain [35] and fatigue 
286 [36], whereas others are asymptomatic. Pain and fatigue may impair physical performance 287 unrelated to the conditions of the muscles.

288

289 During the follow-up period the only statistically significant change observed was a $2 \%$ increase 290 291 of CSA of the knee extensors. No statistically significant changes were observed for any of the clinical tests, the fat fractions or the remaining CSAs. This is in contrast to the study by Khan et al and Nunez-Peralta et al who found a $1.3 \%$ and $1.9 \%$ annual increase in the fat fraction in patients receiving ERT, respectively $[15,29]$. In the studies, however, isokinetic dynamometry was not performed which impedes comparison between the studies.

An increase in size of the knee extensors has previously been described in treatment-naive LOPD patients: In two earlier studies by Pichiecchio et al. [37] and Ravaglia et al. [33] the muscle mass increased after only 6 months of ERT, and more recently a similar increase has been observed in the Embassy study [38]. A possible explanation is that a patient who started on ERT also started to be more active. However, this is not the case of our population, where only 3 patients started ERT treatment in relation to study inclusion.

During the follow-up period the mean fat fraction of the muscles remained stable, which is as described in the Embassy study. However, there was a large variation in the relative changes in fat fraction during the follow-up. Similar variations in changes of fat fraction have also been observed in patients with LGMD21 [39]. In contrast, Carlier et al. [14] observed an increase in fat fraction in 23 treated and untreated LOPD patients after only one year, with more rapid muscle degeneration in patients not receiving ERT. Also, despite ERT, Pichiecchio et al. [37] and Ravaglia et al. [33] reported an increased fat fraction of thigh muscles over time by quantitative MRI. Compared to these studies, our population was considerably younger (mean age: 36 years, compared to 49 [14], 61 [37] and 54 [33] years, respectively). As fat replacement is part of the physiological ageing of striated muscles, this may partly explain the conflicting findings on muscle degeneration.

A recent retrospective study by Lollert et al. [32] including 13 patients with LOPD (age $30 \pm 17.4$, range 6-13) determined changes over time in intramuscular fat in the psoas major muscle and the paraspinal muscles. After 39 months, the fat fraction had increased in the psoas major 
315 muscle. However, subgroup analysis of 7 subjects, who were followed for a longer time, did not 316 reveal any significant change in fat fraction, indicating the general challenge of receiving 317 statistical power in evaluation of rare disorders. Interestingly, in a newly published study by 318 Rehmann et al, use of diffusion tensor imaging in MRI disclose abnormalities in muscle groups with low fat fraction which remain undetected by more conventional MRI techniques [17].

320

321 Our findings indicate that the contractile CSA more closely relates to the muscle strength of the 322 given muscle as compared to fat fraction, which is in agreement with Carlier et al. [11].

323 Muscle imaging is a valuable tool to evaluate disease status, as it reflects muscle degeneration in 324 a quantitative and objective way. However, due to considerable variations, muscle imaging 325 should be considered as a supplement to clinical tests and questionnaires.

326 There are a number of limitations in our study. First, MR image resolution was set to $1 \times 1 \mathrm{~mm}$ 327 in-plane, which sets the lower boundary for precision of the analysis and thereby the sensitivity 328 to detect small changes. However, we did use DIXON over T1 weighed images, thereby, applying 329 a more robust method to alleviate the B0 and B1 field inhomogeneities often present in 3T MR 330 imaging. Second, the limited number of participants does not allow us to perform subgroup 331 analysis, e.g. drug naïve versus treated patients, and patients with low versus high levels of 332 physical activity. Furthermore, not all patients performed all muscles tests which further lower 333 the number of subjects in some analyses. As mentioned, the number of participants is a limiting 334 factor, leading to indication of trends rather than showing statistical differences. The expected 335 difference can be indicated by evaluating results from studies with 41 LOPD (13 in follow up) [32] and 32 LOPD (22 ERT, 10 HyperCKemia) [40] differences measured from MRI segmented muscles.

337 Our study is also limited by a large variation in patient characteristics, including considerable 338 differences in clinical severity and a large age span (19-62 years), which makes subgroup analysis challenging. Due to the low prevalence of LOPD more detailed studies with stratification will only

340 be possible in multicentre studies with standardized MRI protocols, as proposed by Hollingsworth

341 et al. [41]. This will furthermore strengthen the power of the statistical analysis and thereby 342 providing a more precise view of muscle involvement in LOPD. 


\section{5. Conclusion}

344 Contractile CSA closely correlates to muscle strength in patients with LOPD. The average fat ratio 345 of the thigh and hip muscles did not correlate to the 6MWT or the sum rank-score from 346 conventional clinical tests. During the follow-up period the contractile CSA of the knee extensors 347 increased by $2 \%$, whereas, fat fraction of the hip and thigh muscles as well as functional tests 348 remained unchanged. 


\section{References}

[1] R. Hirschhorn, A.J.J. Reuser, ., in: McGraw-Hill (Ed.), Metab. Mol. Bases Inherit. Dis., 8th ed., Scriver, C R Beaudet, A L Sly, WS Valle, D, NY, 2001: pp. 3389-3420.

[2] P.S. Kishnani, W.-L. Hwu, H. Mandel, M. Nicolino, F. Yong, D. Corzo, A retrospective, multinational, multicenter study on the natural history of infantile-onset Pompe disease, J. Pediatr. 148 (2006) 671-676.e2. https://doi.org/10.1016/J.JPEDS.2005.11.033.

[3] A.T. Van Der Ploeg, A.J.J. Reuser, Lysosomal Storage Disease 2 Pompe's disease, Lancet. 372 (2008) 1342-1353. https://doi.org/10.1016/S0140-6736(08)61555-X.

[4] R.-Y. Carlier, P. Laforet, C. Wary, D. Mompoint, K. Laloui, N. Pellegrini, D. Annane, P.G. Carlier, D. Orlikowski, Whole-body muscle MRI in 20 patients suffering from late onset Pompe disease: Involvement patterns., Neuromuscul. Disord. 21 (2011) 791-9. https://doi.org/10.1016/j.nmd.2011.06.748.

[5] M.L.C. Hagemans, L.P.F. Winkel, P.A. Van Doorn, W.J.C. Hop, M.C.B. Loonen, A.J.J. Reuser, A.T. Van der Ploeg, Clinical manifestation and natural course of late-onset Pompe's disease in 54 Dutch patients, Brain. 128 (2005) 671-677.

[6] D. Güngör, J.M. de Vries, W.C.J. Hop, A.J.J. Reuser, P.A. van Doorn, A.T. van der Ploeg, M.L.C. Hagemans, Survival and associated factors in 268 adults with Pompe disease prior to treatment with enzyme replacement therapy, Orphanet J. Rare Dis. 6 (2011) 34. https://doi.org/10.1186/1750-1172-6-34.

[7] J. Chan, A.K. Desai, Z.B. Kazi, K. Corey, S. Austin, L.D. Hobson-Webb, L.E. Case, H.N. Jones, P.S. Kishnani, The emerging phenotype of late-onset Pompe disease: A systematic literature review, Mol. Genet. Metab. . 120 (2017) 163-172. https://doi.org/10.1016/j.ymgme.2016.12.004.

[8] A.T. van der Ploeg, M.E. Kruijshaar, A. Toscano, P. Laforêt, C. Angelini, R.H. Lachmann, S.I. Pascual Pascual, M. Roberts, K. Rösler, T. Stulnig, P.A. van Doorn, P.Y.K. Van den Bergh, J. Vissing, B. Schoser, B. Bembi, A. Broomfield, M. Boentert, C. Desnuelle, O. Findling, A. Hahn, J. Díaz-Manera, T. Hundsberger, C. Kornblum, F. Labarthé, P. Laforet, K.E. Mengel, T. Mongini, W. Muller-Felber, G. Parenti, W.P. Pijnappel, N. Preisler, S. Sacconi, B. Talim, M. Tardieu, N.A.M.E. van der Beek, S. Wenninger, European consensus for starting and stopping enzyme replacement therapy in adult patients with Pompe disease: a 10-year experience, Eur. J. Neurol. 24 (2017) 768-e31. https://doi.org/10.1111/ene.13285.

[9] B. Schoser, P. Laforêt, M.E. Kruijshaar, A. Toscano, P.A. Van Doorn, A.T. Van Der Ploeg, 208th ENMC International Workshop: Formation of a European Network to develop a European data sharing model and treatment guidelines for Pompe disease Naarden, The Netherlands, 26-28 September 2014, Neuromuscul. Disord. 25 (2015) 674-678. https://doi.org/10.1016/j.nmd.2015.04.006.

[10] V. Kalia, D.G. Leung, D.B. Sneag, F. Del Grande, J.A. Carrino, Advanced MRI Techniques for Muscle Imaging, Semin Musculoskelet Radiol. 21 (2017) 459-469. https://doi.org/10.1055/s-0037-1604007.

[11] P.G. Carlier, B. Marty, O. Scheidegger, P. Loureiro de Sousa, P.-Y. Baudin, E. Snezhko, D. Vlodavets, Skeletal Muscle Quantitative Nuclear Magnetic Resonance Imaging and Spectroscopy as an Outcome Measure for Clinical Trials, J. Neuromuscul. Dis. 3 (2016) 128. https://doi.org/10.3233/JND-160145. 
393

394

395

396

397

398

399

400

401

402

403

404

405

406

407

408

409

410

411

412

413

414

415

416

417

418

419

420

421

422

423

424

425

426

427

428

429

430

431

432

433

434

435

436

[12] J. Burakiewicz, C.D.J. Sinclair, D. Fischer, G.A. Walter, H.E. Kan, K.G. Hollingsworth, Quantifying fat replacement of muscle by quantitative MRI in muscular dystrophy, J. Neurol. 264 (2017) 2053-2067. https://doi.org/10.1007/s00415-017-8547-3.

[13] D. Fischer, U. Bonati, M.P. Wattjes, Recent developments in muscle imaging of neuromuscular disorders, Curr. Opin. Neurol. 29 (2016) 614-620. https://doi.org/10.1097/WCO.0000000000000364.

[14] P.G. Carlier, N. Azzabou, P.L. de Sousa, A. Hicks, J.-M. Boisserie, A. Amadon, R.-Y. Carlier, C. Wary, D. Orlikowski, P. Laforêt, Skeletal muscle quantitative nuclear magnetic resonance imaging follow-up of adult Pompe patients, J. Inherit. Metab. Dis. 38 (2015) 565-572. https://doi.org/10.1007/s10545-015-9825-9.

[15] A.A. Khan, T. Boggs, M. Bowling, S. Austin, M. Stefanescu, L. Case, P.S. Kishnani, Wholebody magnetic resonance imaging in late-onset Pompe disease: Clinical utility and correlation with functional measures, J. Inherit. Metab. Dis. (2019) 549-557. https://doi.org/10.1002/jimd.12190.

[16] S. Figueroa-Bonaparte, S. Segovia, J. Llauger, I. Belmonte, I. Pedrosa, A. Alejaldre, M. Mayos, G. Suárez-Cuartín, E. Gallardo, I. Illa, J. Díaz-Manera, S.P.S. Group, Muscle MRI Findings in Childhood/Adult Onset Pompe Disease Correlate with Muscle Function, PLoS One. 11 (2016) e0163493. https://doi.org/10.1371/journal.pone.0163493.

[17] R. Rehmann, M. Froeling, M. Rohm, J. Forsting, R.A. Kley, T. Schmidt-Wilcke, N. Karabul, C.H. Meyer-Frießem, J. Vollert, M. Tegenthoff, M. Vorgerd, L. Schlaffke, Diffusion tensor imaging reveals changes in non-fat infiltrated muscles in late onset Pompe disease, Muscle and Nerve. 62 (2020) 541-549. https://doi.org/10.1002/mus.27021.

[18] Y. Barnouin, G. Butler-Browne, T. Voit, D. Reversat, N. Azzabou, G. Leroux, A. Behin, J.S. McPhee, P.G. Carlier, J.-Y. Hogrel, Manual segmentation of individual muscles of the quadriceps femoris using MRI: a reappraisal., J. Magn. Reson. Imaging. 40 (2014) 239-47. https://doi.org/10.1002/jmri.24370.

[19] T. Harbo, J. Brincks, H. Andersen, Maximal isokinetic and isometric muscle strength of major muscle groups related to age, body mass, height, and sex in 178 healthy subjects, Eur. J. Appl. Physiol. 112 (2012) 267-275. https://doi.org/10.1007/s00421-011-1975-3.

[20] P.. Quanjer, G.J. Tammeling, J.E. Cotes, O.F. Pedersen, R. Peslin, J.-C. Yernault, Lung volumes and forced ventilatory flows, Eur. Respir. J. 6 (1993) 5 LP -40.

[21] A. Pichiecchio, C. Uggetti, S. Ravaglia, M.G. Egitto, M. Rossi, G. Sandrini, C. Danesino, Muscle MRI in adult-onset acid maltase deficiency., Neuromuscul. Disord. 14 (2004) 515. http://www.ncbi.nlm.nih.gov/pubmed/22595200.

[22] J.Y. Hogrel, Y. Barnouin, N. Azzabou, G. Butler-Browne, T. Voit, A. Moraux, G. Leroux, A. Behin, J.S. McPhee, P.G. Carlier, NMR imaging estimates of muscle volume and intramuscular fat infiltration in the thigh: variations with muscle, gender, and age, Age (Omaha). 37 (2015) 1-11. https://doi.org/10.1007/s11357-015-9798-5.

[23] J.M. Morrow, C.D.J. Sinclair, A. Fischmann, P.M. Machado, M.M. Reilly, T.A. Yousry, J.S. Thornton, M.G. Hanna, MRI biomarker assessment of neuromuscular disease progression: a prospective observational cohort study., Lancet. Neurol. 15 (2016) 65-77. https://doi.org/10.1016/S1474-4422(15)00242-2.

[24] J.J. Horvath, S.L. Austin, L.E. Case, K.B. Greene, H.N. Jones, B.J. Soher, P.S. Kishnani, M.R. Bashir, Correlation Between Quantitative Whole-Body Muscle Magnetic Resonance 
437

438

439

440

441

442

443

444

445

446

447

448

449

450

451

452

453

454

455

456

457

458

459

460

461

462

463

464

465

466

467

468

469

470

471

472

473

474

475

476

477

478

479

480

Imaging And Clinical Muscle Weakness In Pompe Disease, Muscle Nerve. 51 (2015) 722730. https://doi.org/10.1002/mus.24437.

[25] B.H. Wokke, J.C. van den Bergen, M.J. Versluis, E.H. Niks, J. Milles, A.G. Webb, E.W. van Zwet, A. Aartsma-Rus, J.J. Verschuuren, H.E. Kan, Quantitative MRI and strength measurements in the assessment of muscle quality in Duchenne muscular dystrophy, Neuromuscul. Disord. 24 (2014) 409-416. https://doi.org/https://doi.org/10.1016/j.nmd.2014.01.015.

[26] A. Alejaldre, J. Díaz-Manera, S. Ravaglia, E.C. Tibaldi, F. D’Amore, G. Morís, N. Muelas, J.J. Vílchez, A. García-Medina, M. Usón, F.A. Martínez García, I. Illa, A. Pichiecchio, Trunk muscle involvement in late-onset Pompe disease: Study of thirty patients, Neuromuscul. Disord. 22 (2012) S148-S154. https://doi.org/10.1016/j.nmd.2012.05.011.

[27] K. Mul, S.C.C. Vincenten, N.C. Voermans, R.J.L.F. Lemmers, P.J. van der Vliet, S.M. van der Maarel, G.W. Padberg, C.G.C. Horlings, B.G.M. van Engelen, Adding quantitative muscle MRI to the FSHD clinical trial toolbox, Neurology. 89 (2017) 2057-2065. https://doi.org/10.1212/WNL.0000000000004647.

[28] G. Andersen, J.R. Dahlqvist, C.R. Vissing, K. Heje, C. Thomsen, J. Vissing, MRI as outcome measure in facioscapulohumeral muscular dystrophy: 1-year follow-up of 45 patients, J. Neurol. 264 (2017) 438-447. https://doi.org/10.1007/s00415-016-8361-3.

[29] C. Nuñez-Peralta, J. Alonso-Pérez, J. Llauger, S. Segovia, P. Montesinos, I. Belmonte, I. Pedrosa, E. Montiel, A. Alonso-Jiménez, J. Sánchez-González, A. Martínez-Noguera, I. Illa, J. Díaz-Manera, Follow-up of late-onset Pompe disease patients with muscle magnetic resonance imaging reveals increase in fat replacement in skeletal muscles, J. Cachexia. Sarcopenia Muscle. (2020). https://doi.org/10.1002/jcsm.12555.

[30] R.J. Maughan, J.S. Watson, J. Weir, Strength and cross-sectional area of human skeletal muscle., J. Physiol. 338 (1983) 37-49.

[31] D. Fischer, P. Hafner, D. Rubino, M. Schmid, C. Neuhaus, H. Jung, O. Bieri, T. Haas, M. Gloor, A. Fischmann, U. Bonati, The 6-minute walk test, motor function measure and quantitative thigh muscle MRI in Becker muscular dystrophy: A cross-sectional study, Neuromuscul. Disord. 26 (2016) 414-422. https://doi.org/http://dx.doi.org/10.1016/j.nmd.2016.04.009.

[32] A. Lollert, C. Stihl, A.M. Hötker, E. Mengel, J. König, K. Laudemann, S. Gökce, C. Düber, G. Staatz, Quantification of intramuscular fat in patients with late-onset Pompe disease by conventional magnetic resonance imaging for the long-term follow-up of enzyme replacement therapy, PLoS One. 13 (2018) 1-15. https://doi.org/10.1371/journal.pone.0190784.

[33] S. Ravaglia, A. Pichiecchio, M. Ponzio, C. Danesino, K. Saeidi Garaghani, G.U. Poloni, A. Toscano, A. Moglia, A. Carlucci, P. Bini, M. Ceroni, S. Bastianello, Changes in skeletal muscle qualities during enzyme replacement therapy in late-onset type II glycogenosis: temporal and spatial pattern of mass vs. strength response., J. Inherit. Metab. Dis. 33 (2010) 737-45. https://doi.org/10.1007/s10545-010-9204-5.

[34] L. Harlaar, J.Y. Hogrel, B. Perniconi, M.E. Kruijshaar, D. Rizopoulos, N. Taouagh, A. Canal, E. Brusse, P.A. van Doorn, A.T. van der Ploeg, P. Laforêt, N.A.M.E. van der Beek, Large variation in effects during 10 years of enzyme therapy in adults with Pompe disease, Neurology. 93 (2019) e1756-e1767. https://doi.org/10.1212/WNL.0000000000008441. 
481

482

483

484

485

486

487

488

489

490

491

492

493

494

495

496

497

498

499

500

501

502

503

504

505

506

507

508

509

510

511

512

513

514

515

516

517

518

519

520

521

[35] D. Güngör, A.K. Schober, M.E. Kruijshaar, I. Plug, N. Karabul, M. Deschauer, P.A. van Doorn, A.T. van der Ploeg, B. Schoser, F. Hanisch, Pain in adult patients with Pompe disease, Mol. Genet. Metab. . 109 (2013) 371-376. https://doi.org/10.1016/j.ymgme.2013.05.021.

[36] M.L.C. Hagemans, S.P.M. van Schie, A.C.J.W. Janssens, P.A. van Doorn, A.J.J. Reuser, A.T. van der Ploeg, Fatigue: an important feature of late-onset Pompe disease, J. Neurol. 254 (2007) 941-945. https://doi.org/10.1007/s00415-006-0434-2.

[37] a Pichiecchio, G.U. Poloni, S. Ravaglia, M. Ponzio, G. Germani, D. Maranzana, a Costa, a Repetto, E. Tavazzi, C. Danesino, a Moglia, S. Bastianello, Enzyme replacement therapy in adult-onset glycogenosis II: is quantitative muscle MRI helpful?, Muscle Nerve. 40 (2009) 122-5. https://doi.org/10.1002/mus.21304.

[38] A. van der Ploeg, P.G. Carlier, R.-Y. Carlier, J.T. Kissel, B. Schoser, S. Wenninger, A. Pestronk, R.J. Barohn, M.M. Dimachkie, O. Goker-Alpan, T. Mozaffar, L.D.M. Pena, Z. Simmons, V. Straub, M. Guglieri, P. Young, M. Boentert, P.-Y. Baudin, S. Wens, R. Shafi, C. Bjartmar, B.L. Thurberg, Prospective exploratory muscle biopsy, imaging, and functional assessment in patients with late-onset Pompe disease treated with alglucosidase alfa: The EMBASSY Study., Mol. Genet. Metab. 119 (2016) 115-23. https://doi.org/10.1016/j.ymgme.2016.05.013.

[39] T.A. Willis, K.G. Hollingsworth, A. Coombs, M.L. Sveen, S. Andersen, T. Stojkovic, M. Eagle, A. Mayhew, P.L. de Sousa, L. Dewar, J.M. Morrow, C.D.J. Sinclair, J.S. Thornton, K. Bushby, H. Lochmüller, M.G. Hanna, J.Y. Hogrel, P.G. Carlier, J. Vissing, V. Straub, Quantitative Muscle MRI as an Assessment Tool for Monitoring Disease Progression in LGMD2I: A Multicentre Longitudinal Study, PLoS One. 8 (2013). https://doi.org/10.1371/journal.pone.0070993.

[40] S. Figueroa-Bonaparte, J. Llauger, S. Segovia, I. Belmonte, I. Pedrosa, E. Montiel, P. Montesinos, J. Sánchez-González, A. Alonso-Jiménez, E. Gallardo, I. Illa, M.A. BarbaRomero, J. Barcena, P. Carbonell, M.R. Carzorla, C. Creus, J. Coll-Cantí, M. Díaz, C. Domínguez, R. Fernández-Torrón, M.J. García-Antelo, J.M. Grau, A.L. De Munáin, F.A. Martínez-García, Y. Morgado, A. Moreno, G. Morís, M.A. Muñoz-Blanco, A. Nascimento, J.L. Parajua, A. Robledo-Strauss, Í. Rojas-Marcos, J.A. Salazar, M. Usón, J. Díaz-Manera, Quantitative muscle MRI to follow up late onset Pompe patients: A prospective study, Sci. Rep. 8 (2018) 1-11. https://doi.org/10.1038/s41598-018-29170-7.

[41] K.G. Hollingsworth, P.L. de Sousa, V. Straub, P.G. Carlier, Towards harmonization of protocols for MRI outcome measures in skeletal muscle studies: consensus recommendations from two TREAT-NMD NMR workshops, 2 May 2010, Stockholm, Sweden, 1-2 October 2009, Paris, France., Neuromuscul. Disord. 22 Suppl 2 (2012) S5467. https://doi.org/10.1016/j.nmd.2012.06.005.

Peer] reviewing PDF | (2020:08:52347:1:2:NEW 12 Jan 2021) 


\section{Highlights}

523

524 1. Contractile CSA of lower extremities muscle groups correlates to muscle strength.

5252 . The fat ratio of the thigh and hip muscles did not correlate to clinical tests.

526 3. At follow-up the contractile CSA of the knee extensors had increased by $2 \%$.

527 4. Fat fraction of the other muscle groups and all functional tests remained unchanged.

528

529

530

531

532

533

534

535

536

537

538

539

540

541

542

543

544

545 
Figure 1

Flow chart of late onset pompe disease and healthy control subjects participating in the study.

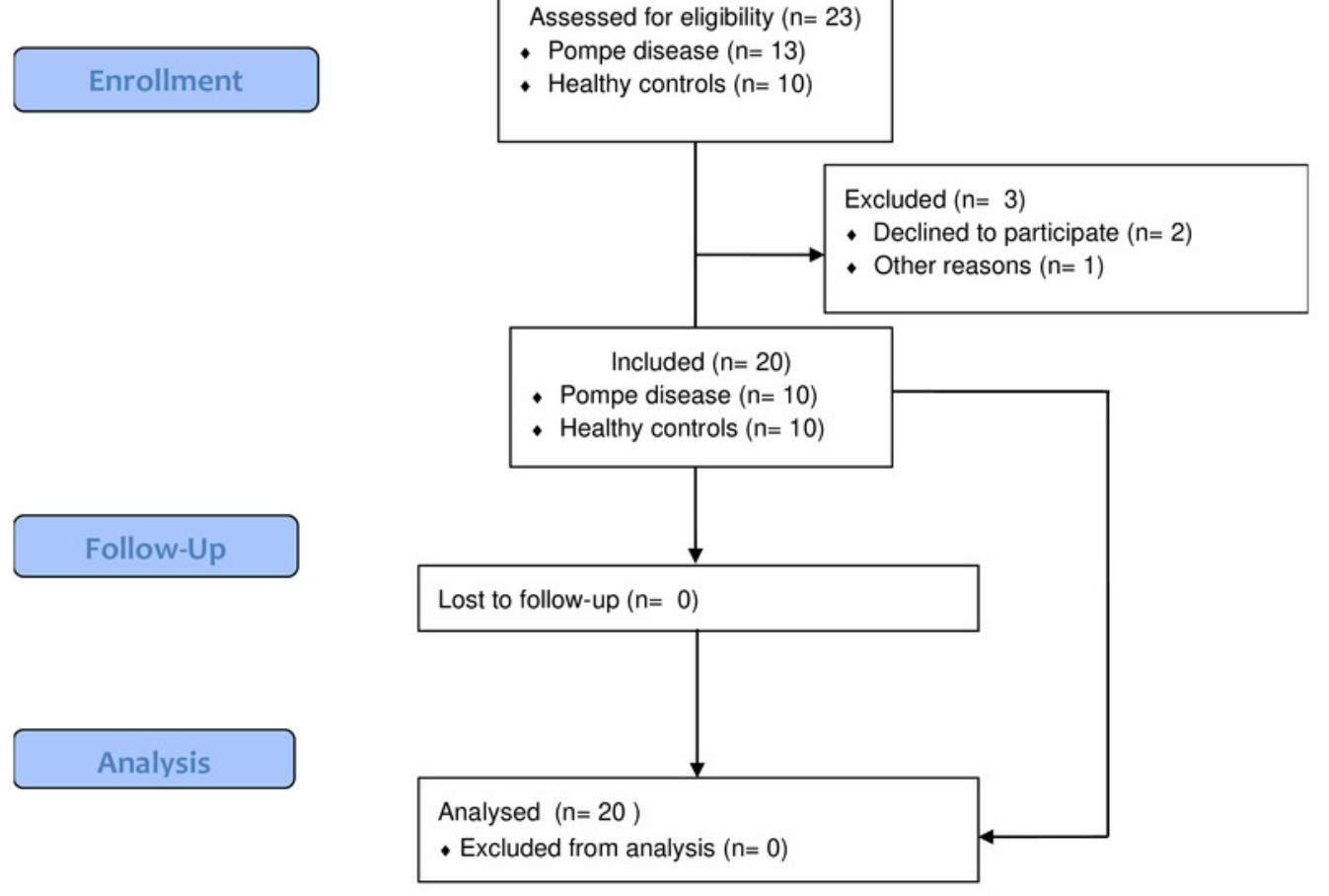




\section{Figure 2}

Illustration of muscle segmentation in the opposed phase DIXON MR images of the muscles of hip flexor/extensor and knee flexors/extensors, corresponding to the following individual muscles:

M. iliopsoas, M. gluteus maximus, $M$. rectus femoris, $M$. sartorius, $M$. vastus medialis, $M$. vastus intermedius, $M$. vastus lateralis, $M$. biceps femoris, $M$. semimembranosus, $M$. semitendinosus. The images are shown at comparable slices of A) a healthy subject, B) segmentation of the healthy subject muscles', and C) a late onset Pompe subject.

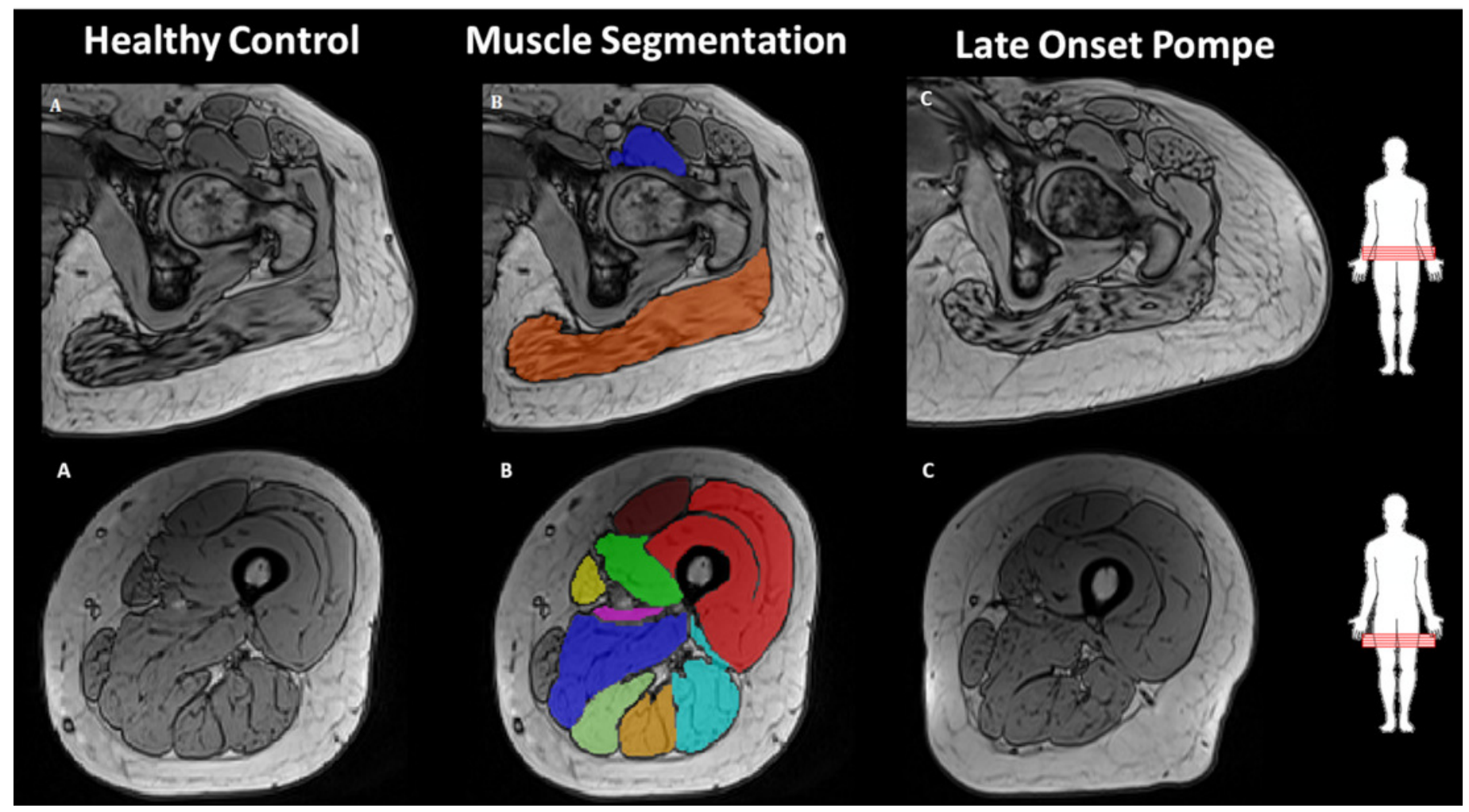


Figure 3

Dot plot illustrating the fat fraction of each muscle in patients (circles) and controls (triangles).

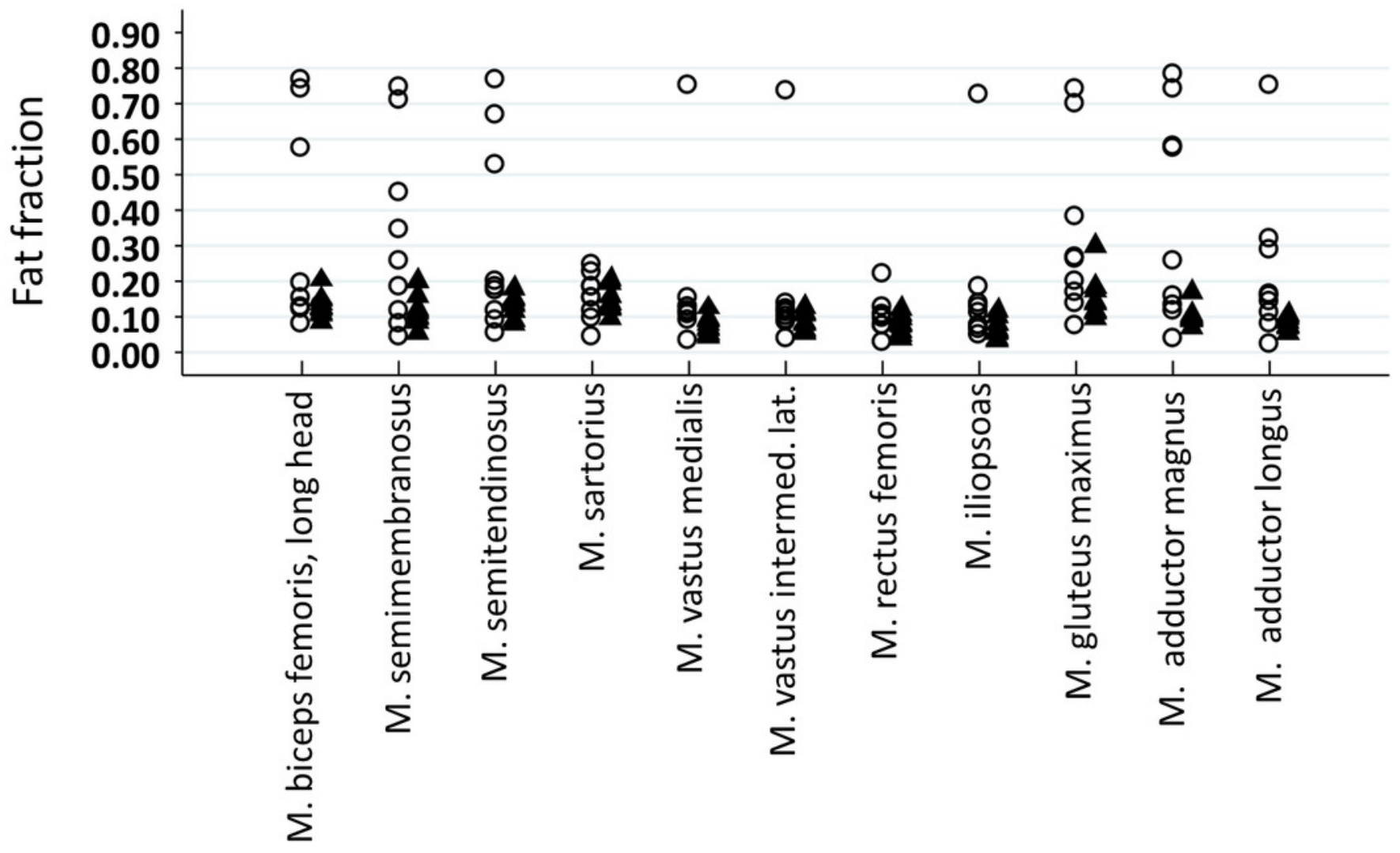

- Healthy controls $\bigcirc$ Patients with LOPD 
Figure 4

Linear regression analysis of muscle strength and contractile CSA of the knee flexors (a) and knee extensors (b) in patients with LOPD (circle) and controls (triangle).
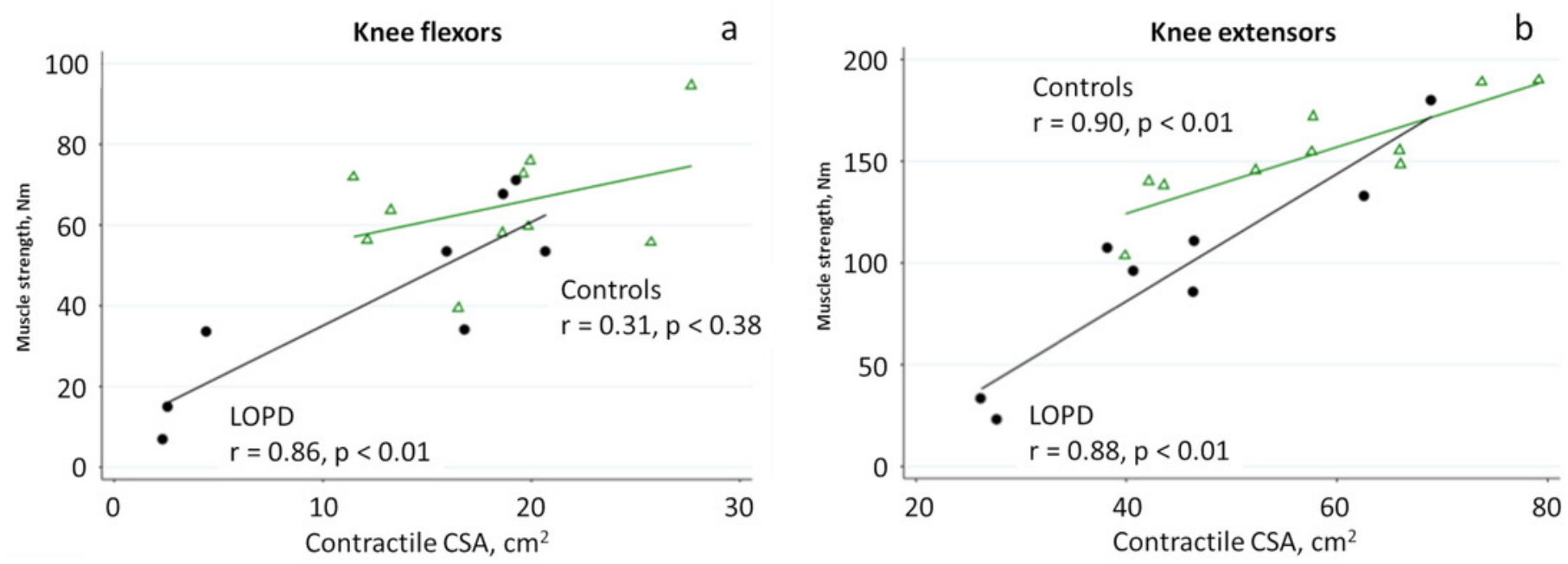
Table $\mathbf{1}$ (on next page)

Subjects' demographics

(Values are given in mean values and range). 
1 Table 1 - Subjects' demographics

\begin{tabular}{lll}
\hline & LOPD & Controls \\
\hline Number of subjects & 10 & 10 \\
\hline Female (\%) & $5(50)$ & $5(50)$ \\
\hline Age (years) & $36(19-62)$ & $38(21-62)$ \\
\hline Height $(\mathrm{cm})$ & $178(161-193)$ & $174(161-$ \\
& & $180)$ \\
\hline Weight $(\mathrm{kg})$ & $72.4(58-102)$ & $72.9(59-92)$
\end{tabular}

2 (Values are given in mean values and range).

3 


\section{Table 2 (on next page)}

Clinical characteristics of the late onset pompe disease patients

During the study period patient 1-9 received alglucosidase alfa. Patient 10 received avalglucosidase alfa (neoGAA). ERT: Enzyme replacement therapy 
Table 2 - Clinical characteristics of the LOPD patients

Patient Sex Age Year of Start Aids Gene mutation
diagnosis on
ERT

1

2

3

4

5

6

M $\quad 44 \quad 2010$

$$
\text { F } \quad 40 \quad 2015
$$

M $\quad 42 \quad 2015$

2015

2011

2007

night $13 T>A$

B-pap at GAA-mutation: c*-32-

GAA-mutation:

c. $1003 \mathrm{G}>\mathrm{A}$

$\div \quad$ GAA-mutation: c-32-

13T $>A$

GAA-mutation:

c.307T>G;pC103G

$\div \quad$ GAA-mutation: c.32-

$2015 \quad 13 T>G$,

Homozygous

$\div \quad$ GAA-mutation: c.32-

$13 T>G$

GAA-mutation: c.525 del,

p. Glu176Argfs*45

$\div \quad$ GAA-mutation: c.32-

13T $>A$

2011 GAA-mutation:

c. $2331+2 T>G$

Wheel GAA-mutation: c-32-

chair for $13 T>G$

2016 longer GAA-mutation: c-525 del,

distance p. Glu176Argfs*45

$7 \quad F \quad 252013 \quad 2014$ Wheel GAA-mutation: c-32- 
8

9

10 chair for $13 T>G$

longer GAA-mutation: c-525 del,

distance p. Glu176Argfs*45

$\div$

GAA-mutation: c.32-

$13 T>G$

M $\quad 19 \quad 2014 \quad 2015$

GAA-mutation:

C. $1548 \mathrm{G}>\mathrm{A}$

$\div \quad$ GAA-mutation: c.32-

$\begin{array}{llll}F & 34 & 2003 & 2014\end{array}$

$13 T>G$

GAA-mutation: c. $1802 \mathrm{C}>\mathrm{T}$

$\div$

GAA-mutation: IVS1-13T

$>\mathrm{G}$

GAA-mutation: $2.228 \mathrm{~A}>\mathrm{G}$

3 During the study period patient 1-9 received alglucosidase alfa. Patient 10 received

4 avalglucosidase alfa (neoGAA).

5 ERT: Enzyme replacement therapy

6

7

8 


\section{Table 3 (on next page)}

Fat fraction, contractile CSA and muscle strength in patients and in healthy controls.

$* p<0.05, * * p<0.01$. Values are given as mean and standard deviation or median and range. The superscript indicates the number of missing data in the patients group in the given analysis, e.g. fat-fraction ${ }^{1}$ indicates one missing data. Statistically significant differences are presented in bold. 
1 Table 3 - Fat fraction, contractile CSA and muscle strength in patients and 2 in healthy controls.

\begin{tabular}{|c|c|c|c|}
\hline \multirow{26}{*}{ Fat fraction } & & Patients & Controls \\
\hline & Knee-extensors ${ }^{1}$ & $0.12(0.03-0.49)$ & $0.09 \pm$ \\
\hline & M. sartorius ${ }^{1}$ & $0.16 \pm 0.06$ & 0.02 \\
\hline & M. rectus femoris ${ }^{1}$ & $0.10 \pm 0.05$ & $0.14 \pm$ \\
\hline & M. vastus medialis ${ }^{1}$ & $0.11(0.03-0.75)$ & 0.03 \\
\hline & M. vastus lateralis. et & $0.11(0.04-0.73)$ & $0.08 \pm$ \\
\hline & intermedius ${ }^{1}$ & $0.21(0.06-$ & 0.03 \\
\hline & Knee-flexors ${ }^{1}$ & $0.75) *$ & $0.07 \pm$ \\
\hline & M. biceps femoris, caput & $0.15(0.08-$ & 0.02 \\
\hline & longum $^{1}$ & $0.76) *$ & $0.08 \pm$ \\
\hline & M. semimembranosus ${ }^{1}$ & $0.32 \pm 0.26 *$ & 0.03 \\
\hline & M. semitendinosus ${ }^{1}$ & $0.18(0.05-$ & $0.12 \pm$ \\
\hline & M. adductor magnus ${ }^{1}$ & $0.77) *$ & 0.03 \\
\hline & M. adductor longus ${ }^{1}$ & $0.37 \pm 0.29 *$ & $0.12 \pm$ \\
\hline & M. gluteus maximus ${ }^{1}$ & $0.16(0.02-$ & 0.02 \\
\hline & M. iliopsoas ${ }^{1}$ & $0.75) *$ & $0.11 \pm$ \\
\hline & & $0.32 \pm 0.24 *$ & 0.04 \\
\hline & & $0.11(0.05-$ & $0.11 \pm$ \\
\hline & & $0.73) *$ & 0.03 \\
\hline & & & 0.10 \\
\hline & & & \pm 0.03 \\
\hline & & & $0.08 \pm$ \\
\hline & & & 0.02 \\
\hline & & & $0.15 \pm$ \\
\hline & & & 0.06 \\
\hline & & & $0.07 \pm$ \\
\hline & & & 0.03 \\
\hline
\end{tabular}


Contractile

CSA

$\left(\mathrm{cm}^{2}\right)$
Knee-extensors ${ }^{1}$

M. sartorius ${ }^{1}$

M. rectus femoris ${ }^{1}$

M. vastus medialis ${ }^{1}$

M. vastus lateralis et

intermedius ${ }^{1}$

Knee-flexors ${ }^{1}$

M. biceps femoris, caput longum ${ }^{1}$

M. semimembranosus ${ }^{1}$

M. semitendinosus ${ }^{1}$

M. adductor magnus ${ }^{1}$

M. adductor longus ${ }^{1}$

M. gluteus maximus ${ }^{1}$

M. iliopsoas ${ }^{1}$
$45.53 \pm 14.38 * 57.84 \pm$

$2.81 \pm 1.10$

$7.15 \pm 2.01$

$4.67 \pm 2.66 * *$

$30.79 \pm 12.05 *$

16.83 (2.34-

20.70)*

$6.56(0.66-$

8.49)*

$2.19 \pm 1.57 *$

$5.54 \pm 3.48$

$7.87 \pm 6.83 * *$

$3.11 \pm 1.98$

$28.50 \pm 14.76 *$

$5.98 \pm 3.28$

15.51

$40.61 \pm$

9.41

$18.49 \pm$

5.41

$20.72 \pm$

5.02
$2.54 \pm 0.67$

$6.02 \pm 1.76$

$9.26 \pm 2.81$

$7.66 \pm 2.51$

$2.64 \pm 2.1$ :

$7.03 \pm 1.84$

$4.56 \pm 1.36$

$39.49 \pm$

8.43

$6.68 \pm 1.27$

Muscle

Knee-extensors ${ }^{1}$

103.2 土 52.3**

$46.0 \pm 25.2$

$153.4 \pm$

Knee-flexors ${ }^{1}$

Hip extensors ${ }^{2}$

Hip flexors 4

Foot flexors ${ }^{1}$

Foot extensors ${ }^{1}$

Shoulder abductor ${ }^{1}$

Shoulder adductor ${ }^{1}$

Elbow flexors ${ }^{1}$

Elbow extensors ${ }^{1}$

Hand flexors ${ }^{1}$
$62.8 \pm 42.3 * \quad 64.5 \pm$

$77.0 \pm 35.1 * * \quad 12.6$

$52.4 \pm 13.5$

$20.3 \pm 15.2$

$139.8 \pm$

25.5

$37.8 \pm 15.7$

$120.6 \pm$

$43.4 \pm 23.1 * \quad 29.7$

$33.6 \pm 11.7 *$

$62.0 \pm$

$33.2 \pm 11.5$

11.3

$16.6 \pm 7.1$ 
Hand extensors
$8.8 \pm 4.2$
14.7

$44.8 \pm$

12.9

$62.1 \pm$

\section{2}

$40.6 \pm$

\section{2}

$35.8 \pm 8.4$

$14.9 \pm 4.1$

$9.6 \pm 1.7$

$3 * \mathrm{p}<0.05, * * \mathrm{p}<0.01$.

4 Values are given as mean and standard deviation or median and range.

5 The superscript indicates the number of missing data in the patients group in the given

6 analysis, e.g. fat-fraction ${ }^{1}$ indicates one missing data. Statistically significant differences are 7 presented in bold.

8

9

10

11 


\section{Table 4 (on next page)}

Correlations between muscle strength and the corresponding MRI findings from the muscles (contractile CSA and fatfraction) and between the fatfraction and the clinical tests (sum rank score and 6MWT).

$p<0.05, * * p<0.01$. Analysis was done by Spearman's correlation. The superscript indicates the number of missing data in the patients group in the given analysis, e.g. knee flexors ${ }^{2}$ indicates missing data from two patients. 
1 Table 4 - Correlations between muscle strength and the corresponding MRI

2 findings from the muscles (contractile CSA and fat fraction) and between the

3 fat fraction and the clinical tests (sum rank score and 6MWT).

Patients Controls

Contractile Muscle strength

CSA

\begin{tabular}{|c|c|c|}
\hline Knee flexors ${ }^{2}$ & $\mathbf{r}=0.86 * *$ & $r=0.31$ \\
\hline Knee extensors ${ }^{2}$ & $\mathbf{r}=0.88 * *$ & $\mathbf{r}=0.90 * *$ \\
\hline Hip extensor ${ }^{2}$ & $r=0.83 *$ & $r=0.38$ \\
\hline Hip flexor ${ }^{3}$ & $r=0.29$ & $\mathbf{r}=0.67 * *$ \\
\hline
\end{tabular}

Fat fraction Muscle strength

\begin{tabular}{|c|c|c|}
\hline Knee flexors ${ }^{2}$ & $r=-0.83 *$ & $r=-0.14$ \\
\hline Knee extensors ${ }^{2}$ & $r=-0.43$ & $r=-0.25$ \\
\hline Hip extensor ${ }^{2}$ & $r=-0.68$ & $r=0.07$ \\
\hline Hip flexor ${ }^{3}$ & $r=-0.14$ & $r=0.24$ \\
\hline
\end{tabular}

Total fat Sum rank-score ${ }^{1} \quad r=0.38$

fraction

Total fat $\quad 6 \mathrm{MWT}^{1} \quad r=-0.50$

fraction

$4 \mathrm{p}<0.05, * * \mathrm{p}<0.01$.

5 Analysis was done by Spearman's correlation. The superscript indicates the number of

6 missing data in the patients group in the given analysis, e.g. knee flexors ${ }^{2}$ indicates missing

7 data from two patients.

8 


\section{Table 5 (on next page)}

Clinical test at baseline and at follow-up.

MMT = Manual muscle testing. SF-36 = 36-item short form questionnaire. 6 minute walking test (6MWT) is measured in minutes. 
1 Table 5 - Clinical test at baseline and at follow-up.

6MWT (m)

$\%$ of expected FVC

sitting position

lying position

MMT

SF-36

$\%$ of expected total strength
Baseline

$506.6 \pm 143.89$
Follow-up

$490.3 \pm 164.38$

2

$3 \mathrm{MMT}=$ Manual muscle testing. SF-36 $=36$-item short form questionnaire. 6 minute walking 4 test (6MWT) is measured in minutes.

5 


\section{Table 6(on next page)}

The contractile cross-sectional area (CSA) at baseline and at follow-up.

The mean difference is calculated as follow-up value minus baseline value. Contractile crosssectional area (CSA) is measured in $\mathrm{cm}^{2}$. Values are given in mean and SD; Data without normal distribution are also presented as median and range. 
1 Table 6 - The contractile cross-sectional area (CSA) at baseline and at

2 follow-up.

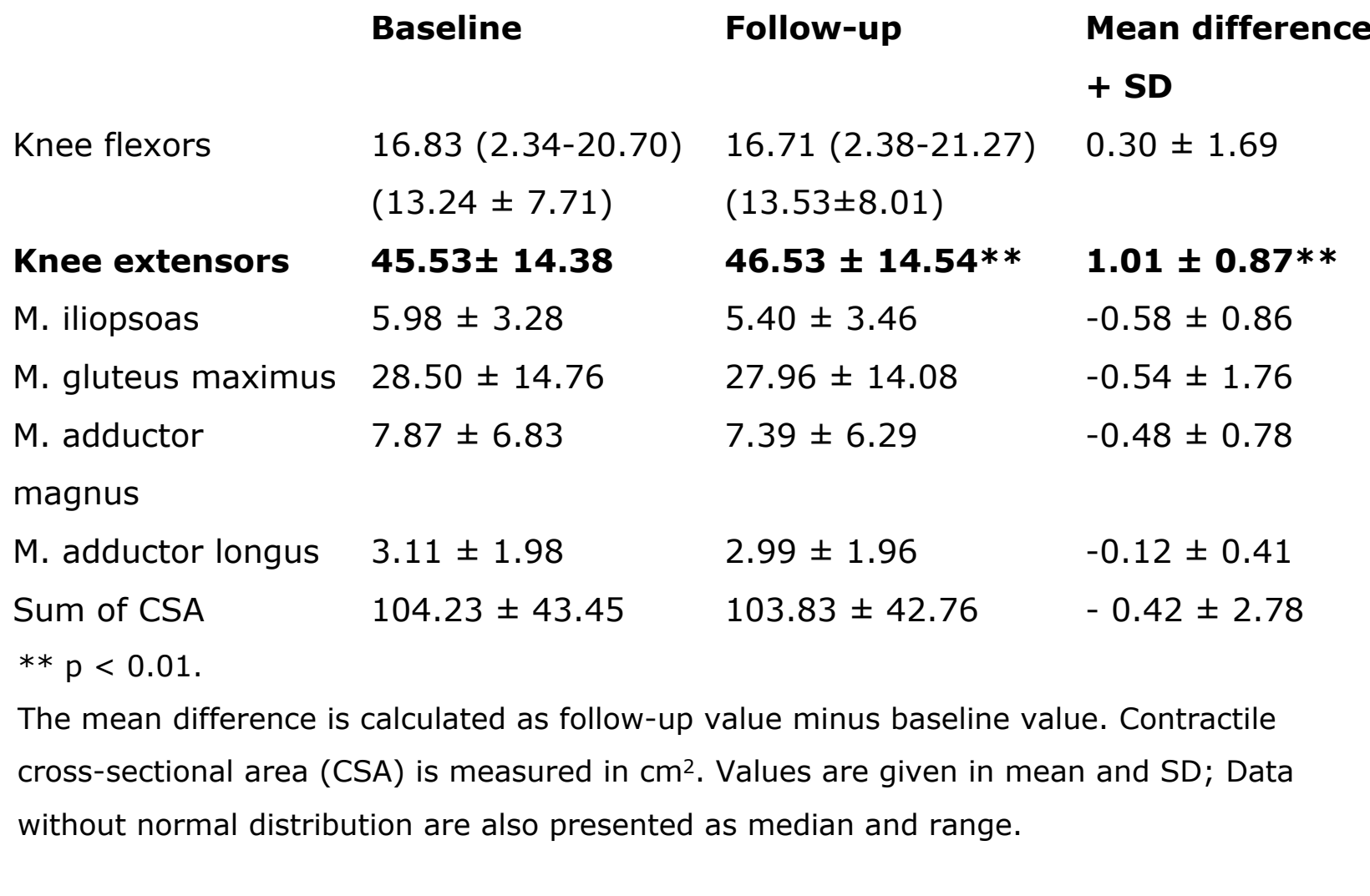




\section{Table 7 (on next page)}

The fat fraction of the late onset Pompe Disease patients at baseline and follow-up.

The mean difference is calculated as follow-up value minus baseline value. Values are given in mean and SD; where data were not normally distributed median and range is given first. 
1 Table 7 -The fat fraction of the late onset Pompe Disease patients at baseline and follow-up.

Fat fraction

Knee flexors

M. semimembranosus

M. semitendinosus

Knee extensors

M. vastus med

(1)

$M$. vastus lateralis et

intermedius

M. sartorius

M. rectus femoris

M. iliopsoas

(1)

M. gluteus maximus

M. adductor magnus

M. adductor longus

Sum of fat fractions
Baseline

$0.21(0.06-0.75)$

$(0.32 \pm 0.27)$

0.15 (0.08 - 0.76)

$(0.32 \pm 0.28)$

$0.32 \pm 0.26$

$0.18(0.05-0.77)$

$(0.31 \pm 0.27)$

0.12 (0.03 - 0.49)

$(0.15 \pm 0.13)$

0.11 (0.03 - 0.75$)$

$(0.18 \pm 0.22)$

0.11 (0.04 - 0.73)

$(0.17 \pm 0.21)$

$0.16 \pm 0.06$

$0.10 \pm 0.05$

$0.11(0.05-0.73)$

$(0.17 \pm 0.21)$

$0.32 \pm 0.24$

$0.37 \pm 0.29$

0.16 (0.02 - 0.75$)$

$(0.23 \pm 0.22)$
Follow-up

$0.18(0.08-0.76)$

$(0.32 \pm 0.28)$

0.14 (0.10-0.76)

$(0.32 \pm 0.29)$

$0.32 \pm 0.26$

0.19 (0.08 - 0.78)

(0.32 \pm 0.28$)$

0.13 (0.07-0.50)

$(0.16 \pm 0.13)$

$0.21 \pm 0.23$

0.10 (0.06 - 0.76)

$0.17 \pm 0.22$

$0.15 \pm 0.06$

$0.09 \pm 0.04$

0.08 (0.05 - 0.69)

$(0.15 \pm 0.20)$

$0.33 \pm 0.24$

$0.38 \pm 0.30$

0.15 (0.06 - 0.73)

$0.22 \pm 0.22$
0.13 (0.07 - 0.78)

Mean difference

+ SD

$0.00 \pm 0.02$

$0.00 \pm 0.02$

$-0.00 \pm 0.03$

$0.01 \pm 0.03$

$0.00 \pm 0.03$

$0.03 \pm 0.07$

$0.00 \pm 0.02$

$-0.01 \pm 0.02$

$-0.01 \pm 0.03$

$-0.02 \pm 0.03$

$0.26 \pm 0.20$
$0.00 \pm 0.03$

$0.01 \pm 0.03$

$-0.00 \pm 0.04$

2 The mean difference is calculated as follow-up value minus baseline value.

3 Values are given in mean and SD; where data were not normally distributed median and range is given first. 